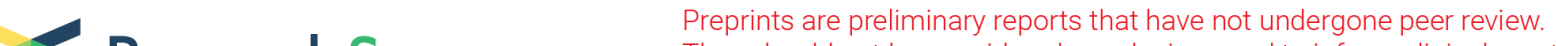 Research Square They should not be considered conclusive, used to inform clinical practice, or referenced by the media as validated information.
}

\section{Long Non-Coding RNA DANCR Participates in the Regulation of Dexamethasone and Inflammation Factors on hASC Proliferation and Migration}

\section{Ran Yan}

Chinese Academy of Medical Sciences \& Peking Union Medical College Plastic Surgery Hospital and Institute

\section{Ping Dong}

Chinese Academy of Medical Sciences \& Peking Union Medical College Plastic Surgery Hospital

\section{Zhigang Yang}

Chinese Academy of Medical Sciences \& Peking Union Medical College Plastic Surgery Hospital and Institute

\section{Rui Cao}

Chinese Academy of Medical Sciences \& Peking Union Medical College Plastic Surgery Hospital and Institue

Xia Liu ( $\square$ liuxia@psh.pumc.edu.cn )

Chinese Academy of Medical Sciences Plastic Surgery Hospital and Institute https://orcid.org/00000003-0832-7772

\section{Ran Xiao}

Chinese Academy of Medical Sciences \& Peking Union Medical College Institute of Biomedical Engineering

\section{Research}

Keywords: DANCR, long non-coding RNA, Dexamethasone, TNF-a, hASC, proliferation, migration

Posted Date: January 15th, 2021

DOl: https://doi.org/10.21203/rs.3.rs-144256/v1

License: (c) (1) This work is licensed under a Creative Commons Attribution 4.0 International License. Read Full License 
3 Long non-coding RNA DANCR participates in the regulation of

4 dexamethasone and inflammation factors on hASC proliferation and

5 migration

Author names and affiliations: Ran Yan ${ }^{1}$, Ping Dong ${ }^{1}$, Zhigang Yang ${ }^{1}$,

Rui $\mathrm{Cao}^{1}, \mathrm{Xia} \mathrm{Liu}^{1 *}$, Ran Xiao ${ }^{1 *}$

91 Research Center of Plastic Surgery Hospital, Chinese Academy of

Medical Sciences, Peking Union Medical College, Beijing, People’s

11 Republic of China;

*Corresponding author:

14 Xia Liu, Professor, liuxia@psh.pumc.edu.cn;

Ran Xiao; Professor, xiaoran@psh.pumc.edu.cn;

Research Center of Plastic Surgery Hospital, Ba-Da-Chu Rd. 33, Beijing,

100144, P.R. China. Telephone: 86-10-88771507; Fax: 86-10-88960373;

\section{Acknowledgments:}

This study was supported by the National Natural Science Foundation of

China (81873666,81871575), and the CAMS Innovation Fund for

22 Medical Sciences (CIFMS, grant no. 2016-I2M-1-017), and Program for 
1 Union Scholars and Innovative Research Team in Peking Union Medical

2 College, and Non-profit Central Research Institute Fund of Chinese

3 Academy of Medical Sciences (2018PT32015).

4

5

\section{Abstract}

Background: Both mesenchymal stem cells (MSCs) and Dexamethasone (Dex) are effective methods to treat inflammatory diseases, and they are likely to be used in combination. The proliferation and migration ability is one of the main biological characteristics of MSCs for repairing. However, the effect of inflammatory factors and Dex on these characteristics of MSCs has not been fully understood. Therefore, this study aimed to determine the role of differentiation antagonizing nonprotein coding RNA (DANCR) in hASC proliferation and migration regulation induced by Dex and inflammatory factors, to clarify the effect and mechanism of glucocorticoids on MSC's characteristics to participate in tissue repair in an inflammatory environment.

Methods: Human adipose derived stem cells (hASCs) were cultured and treated with dexamethasone and inflammation factors, and cell proliferation, migration abilities, and DANCR mRNA expression were detected. Additionally, to determine the roles and mechanisms, DANCR was knockdown or overexpressed before Dex or tumor necrosis factor alpha (TNF- $\alpha$ ) treatments. HASC proliferation was tested by cell 
1 counting kit-8 and cell cycle assay. HASC migration ability was analyzed

2 by a scratching would healing test. Moreover, proliferation and

3 migration-related genes were measured by a real-time quantitative reverse

4 transcription-polymerase chain reaction (qRT-PCR). Nuclear factor-kB

5 (NF-kB) and PI3K-AKT-mTOR pathway proteins were investigated by

6 western blot analysis. All values are expressed as the mean \pm standard

7 error of the mean. The differences between the groups were assessed

8 using a two-tailed Student's t-test.

9 Results: Dex decreased the proliferation and migration of hASCs in a

10 dose-dependent manner. Dex could upregulate the expression of DANCR

11 that inhibited hASC proliferation and migration. Knockdown of DANCR

12 reversed the inhibition of hASC proliferation and migration induced by

Dex. Moreover, DANCR was decreased by inflammatory cytokines, and

overexpression of DANCR alleviated the promotion of hASC proliferation and migration induced by TNF- $\alpha$. Furthermore, mechanistic investigation validated that DANCR was involved in the NF- $\mathrm{kB}$ signaling pathway.

Conclusions: We identified a long non-coding RNA (lncRNA),

DANCR, involved in Dex and inflammation-affected hASC proliferation and migration, thus suggesting that concurrent application of hASCs with steroids should be avoided in clinical settings. DANCR may serve as a

22 promising approach to regulate stem cell characteristics under an 
1 inflamed microenvironment. These findings further enrich our

2 understanding of the functional versatility of lncRNAs in the crosstalk of

3 inflammation conditions and stem cells.

4 Keywords: DANCR; long non-coding RNA; Dexamethasone; TNF- a ;

5 hASC; proliferation; migration

6

7 
1

2 Mesenchymal stem cells (MSCs) are multipotent cells with

3 immunomodulatory functions, which have been being clinically explored

4 as a new treatment for various inflammation or immune-related diseases,

5 including arthritis, graft-versus-host disease, and inflammatory bowel

6 disease, et al. Most preclinical and clinical studies are performed using

7 MSCs derived from adult bone marrow and adipose tissue. The great

8 advantage of human adipose derived stem cells (hASCs) is that they can

9 be isolated in large quantities from very abundant and easily accessible

10 adipose tissue ${ }^{1,2}$.

11 Glucocorticoid therapy is an important approach to managing 12 inflammatory and autoimmune disorders. Dexamethasone (Dex) is a synthetic glucocorticoid hormone that can play an important role in metabolism and immune regulation and is widely used in the treatment of inflammatory or immune-related diseases. Additionally, several studies have verified the impact of Dex on the characteristics of MSCs, including differentiation, proliferation, and immunomodulatory properties ${ }^{3,4}$.

Long noncoding RNAs (lncRNAs), with a size of longer than 200 nucleotides and no protein-coding capacity, have been recognized as important regulators in diverse biological processes, such as transcriptional regulation, stem cell proliferation, migration, and differentiation ${ }^{5,6,7}$. Recent studies also demonstrate that lncRNAs may act as key regulators of 
1 the inflammatory response ${ }^{8,9}$. However, the relationship between lncRNAs

2 and hASC characteristics under inflammatory conditions was not certain.

3 In our research, we found that Dex can increase the expression of

4 differentiation antagonizing non-protein coding RNA (DANCR)

5 expression for the first time. DANCR is expressed and plays important

6 roles in a variety of stem cells ${ }^{10,11,12}$. Our study aims to determine the role

7 of DANCR in hASC proliferation and migration induced by Dex and 8 inflammatory factors, to clarify the effect and mechanism of 9 glucocorticoids on MSC's characteristics to participate in tissue repair in an inflammatory environment.

\section{Materials and Methods}

\section{Human adipose tissue samples collection and cell culture}

The sample collection was approved by the Ethical Committee of Plastic Surgery Hospital (Institute), the Chinese Academy of Medical Sciences, and Peking Union Medical College. Written informed consent for the harvest and use of adipose tissue samples for research purposes was obtained from each patient.

All adipose tissue samples were derived from abdominal liposuction of female donors aged from 25 to 35 years old. All cell-based experiments were repeated in triplicate. We obtained hASCs from the stromal vascular fraction (SVF) that was isolated from adipose tissue by digestion with collagenase. In brief, the adipose tissue was washed with phosphate buffer 
1 saline (PBS) and digested with $0.2 \%(\mathrm{w} / \mathrm{v})$ type I collagenase (Sigma

2 Aldrich, St Louis, MO, USA) at $37^{\circ} \mathrm{C}$ for $30-60$ min with gentle agitation.

3 The suspension was filtered through a 100-mesh filter followed by

4 centrifugation at 1,000 rpm for $10 \mathrm{~min}$, and the final pellet was resuspended

5 in the culture medium.

6 Cells were plated at a density of $1 \times 10^{5} \mathrm{cells} / \mathrm{cm}^{2}$ for culture in

7 Mesenchymal Stem Cell Medium (MSCM, ScienCell, and Carlsbad, CA,

8 USA) containing $10 \%$ fetal bovine serum (FBS, Invitrogen) in a cell

9 incubator at $37^{\circ} \mathrm{C}$ with $5 \% \mathrm{CO}_{2}$. Cells of passage 3 were finally used for

10 all experiments.

11 Inflammatory factors : tumor necrosis factor alpha (TNF- $\alpha$ ), interleukin 1

12 beta (IL-1 $\beta)$, interferon $\gamma($ IFN- $\gamma)$, interleukin 6 (IL-6), interleukin 8(IL-8),

13 monocyte chemoattractant protein-1 (MCP-1), interleukin 13 (IL-13), and

14 interleukin 10 (IL-10) and Dex were added to the hASC culture medium

15 individually, and the expression of DANCR was assessed by real-time

16 quantitative reverse transcription-polymerase chain reaction (qRT-PCR).

17 hASCs treated with $1 \times 10^{-7} \mathrm{~mol} / 1 \mathrm{Dex}$ or $10 \mathrm{ng} / \mathrm{ml} \mathrm{TNF}-\alpha$ were detected for

18 proliferation, cell cycle, and migration ability.

19 Lentivirus vector-mediated DANCR knockdown or overexpression in 20 hASC

21 For the human DANCR (NCBI accession number NR 024031.1) silencing, 22 the hairpin oligonucleotides sequences designed against the DANCR gene 
1 were synthesized, annealed, and ligated into the PLKO.1 cloning vector

2 (Addgene, Cambridge, MA) between the AgeI and EcoRI enzyme sites.

3 The hairpin oligonucleotides sequences of the DANCR shRNA1, shRNA2 4 and scramble were 5' - C C G G G C G T A C T A A C T T G T A G C A 5 A C T C GA G T T G C TA C A A G T TA G T A C G C T T T T T G 6 3' (shDANCR1), 5' -C C G G A T A G G A A A G T G C C T C T A A 7 T A A C T C GAG T T A T T A GA G G CAC T T T C C T A T T T T T $8 \mathrm{~T} \mathrm{G}^{-} 3^{\prime}$ (shDANCR2), 5’ - C C G G C C T A A G G T T A A G T C G C C C T C G C T C GA G C GA G G G C GA C T T A A C C T TA G G T T T T T G - 3' (shScramble). The full length of DANCR was amplified from the cDNA of hASC and inserted into the pCDH-CMV-MCS-EF1CoGFP vector (System Biosciences, Mountain View, CA) between the BamHI and EcoRI enzyme sites. For viral packaging, the vectors were transfected into $293 \mathrm{~T}$ cells together with packaging vectors psPAX2 and pMD2.G (Addgene) by lipoFilter (Hanbio, Shanghai, and People's Republic of China). For cell infection, hASC at passage 3 were used. The infection efficiency was observed after $72 \mathrm{~h}$ through a fluorescence microscope for the green fluorescence protein (GFP) expression. The expression of DANCR in hASCs was detected by qRT-PCR (Roche, LightCycler 480, Switzerland).

\section{Cell proliferation and cell cycle assays}

Cells were seeded into 96 -well plates at a density of $3 \times 10^{3}$ cells per well. 
1 The assay was performed $24 \mathrm{~h}$ after seeding and lasted for 7 days. The cell

2 counting kit-8 (CCK-8) assay (Dojindo, Kumamoto, Japan) was used to

3 measure cell proliferation according to the manufacture's guidelines.

4 Briefly, a $10 \mu \mathrm{l}$ volume of CCK-8 reagents was added for $2 \mathrm{~h}$, and

5 absorbance was tested using a micro-plate reader with $450 \mathrm{~nm}$ wavelengths.

6 Approximately $1 \times 10^{6}$ cells were centrifuged and washed with PBS and

7 then fixed with $70 \%$ ethanol overnight at $-20{ }^{\circ} \mathrm{C}$. Approximately $200 \mathrm{ml}$ of

8 fixed cells and an equal volume of Muse cell cycle reagent (Muse $\bigcirc R C e l l$

9 Cycle Assay Kit, Millipore, Billerica, MA, USA) were mixed and incubated for $30 \mathrm{~min}$ at room temperature in the dark. The cell cycle was analyzed using a Muse Cell Analyzer (Millipore) and the proportion of cells in the G0/G1 phase, S phase, and the G2/M phase was measured.

\section{Cell migration assay}

A scratch would healing test was used to determine the migration ability of hASCs. Briefly, cells were cultured on 6-well plates (Corning, Tewksbury, MA, USA) until reaching 80\%-90\% confluence. Cell scratch wounds were made by scraping the cell layer in each culture plate using a $200-\mu 1$ pipette tip. Then the cells were washed with PBS and cultured in the serum-free medium. Percentage of scratch healing was quantified as (scratch area ${ }_{0 h^{-}}$ scratch area $\left.{ }_{x h}\right) /$ scratch area $0 \mathrm{~h} * 100 \%$.

Real-Time Quantitative Reverse Transcription-Polymerase Chain Reaction (qRT-PCR) 
1 The total RNA was isolated from cells with TRIzol Reagent (Sigma

2 Aldrich) according to the standard procedure. For cDNA synthesis, 2000

3 ng of RNA was reverse transcribed using M-MLV reverse transcriptase

4 (Invitrogen) following the manufacturer's instructions. qRT-PCR was

5 performed using the Fast SYBR Green Master Kit and LightCycler 480

6 system (Roche, Basel, Switzerland) according to the manufacturer's

7 instructions. The expression level of each target gene was normalized to

8 the expression of GAPDH and measured by the comparative $\mathrm{Ct}\left(2^{-\Delta \Delta \mathrm{Ct}}\right)$

9 method. The results were expressed as $\log 10\left(2^{-\Delta \Delta C t}\right)$. Each sample was analyzed in triplicate. Primer sequences were summarized in Table 1.

Table 1. Primer Sequences used for qRT-PCR

\begin{tabular}{|c|c|}
\hline Gene & Primer $\left(5^{\prime}-3^{\prime}\right)$ \\
\hline \multirow[t]{2}{*}{ GAPDH } & F: GCACCGTCAAGGCTGAGAAC \\
\hline & R: TGGTGAAGACGCCAGTGGA \\
\hline \multirow[t]{2}{*}{$c-F o s$} & F: CTTCAACGCAGACTACGAGG \\
\hline & R: GACCGTGGGAATGAAGTTGG \\
\hline \multirow[t]{2}{*}{ c-Jun } & F: CCAACATGCTCAGGGAACAG \\
\hline & R: CTCAAGTCTGTCTCTCTGTG \\
\hline \multirow[t]{2}{*}{ P53 } & F: TGCTCAAGACTGGCGCTAAA \\
\hline & R: CAATCCAGGGAAGCGTGTCA \\
\hline \multirow[t]{2}{*}{$P 21$} & F: GCACTTTGATTAGCAGCGGA \\
\hline & R: GAAAGACAACTACTCCCAGC \\
\hline \multirow[t]{2}{*}{ CHEK 1} & F: AGCGGTTGGTCAAAAGAATG \\
\hline & R: CCCTTAGAAAGCCGGAAGTC \\
\hline \multirow[t]{2}{*}{$D A N C R$} & F: CACAGGAGCTAGAGCAGTGA \\
\hline & R: CAGCTGCATTGAGTTAGCGG \\
\hline \multirow[t]{2}{*}{ CXCR4 } & F:TCTTCCTGCCCACCATCTAC \\
\hline & R:GCGTGATGACAAAGAGGAGG \\
\hline \multirow[t]{2}{*}{$C X C R 7$} & F:CCAGCAGCAGGAAGAAGATG \\
\hline & R:GGGCAGTAGGTCTCATTGTTG \\
\hline
\end{tabular}


1 Cells from each set of experiments were harvested, washed with PBS, and

2 lysed with RIPA lysis buffer (Beyotime, Shanghai, China). Protein

3 concentrations were determined with a BCA Protein Assay Kit (Beyotime).

4 Proteins were separated by $10 \%$ sodium dodecyl sulfate-polyacrylamide

5 gel electrophoresis (SDS-PAGE) and transferred to polyvinylidene fluoride

6 membrane (EMD Millipore), and incubated overnight at $4^{\circ} \mathrm{C}$ with specific

7 antibodies against $\beta$-actin (Abcam, Tokyo, Japan), an inhibitor of nuclear

8 factor kappa-B kinase $\beta$ (IKK $\beta$ ), phospho-IKK $\alpha / \beta$ (Ser176/180), IкB $\alpha$,

9 Phospho-p70 S6 kinase (Thr389), p70 S6 kinase, phospho-AKT (S473),

10 and pan-AKT (all from CST, Danvers, MA, USA). These primary

11 antibodies were diluted at 1:1000. Horseradish-peroxidase-conjugated

12 goat anti-mouse or anti-rabbit or anti-rat secondary antibodies $(1: 100,000$,

13 Santa Cruz, Dallas, Texas, USA) were used and SuperSignal West Pico

14 chemiluminescent substrate (Thermo Fisher) was applied for protein

15 detection. LabWorks v4.6 software (UVP, Inc., Upland, CA, USA) was

applied for quantification of Western blot. The background was subtracted, and the signal of each target was normalized to that of the $\beta$-actin band.

\section{Statistical Analysis}

Experimental data were analyzed with GraphPad Prism 6.0 software (San Diego, CA, USA). All values are expressed as the mean \pm standard error of the mean. The differences between the groups were assessed using a two-tailed Student's t-test. In the case of multiple-group testing, statistical 
1 significance was determined using one-way analysis of variance with

2 Tukey's post hoc multiple comparison test for normally distributed data

3 and otherwise by Kruskal -Wallis test. The results were considered

4 statistically significant at $\mathrm{p}<0.05$.

5 Results

6 Dex decreased the proliferation and migration of hASCs in a dose-

7 dependent manner

8 hASCs were treated with Dex at different concentrations $\left(10^{-6}, 10^{-7}, 10^{-8}\right.$

9 M). First, we evaluated the hASC proliferation capacity using the cck-8 kit.

10 The results showed that Dex significantly inhibited cell proliferation

11 (Figure 1A) and the expression of proliferation-related genes (c-Jun, c-Fos)

12 (Figure 1B) at a concentration of $10^{-6}$ and $10^{-7} \mathrm{M}$. The cell cycle 13 distribution of hASC was determined using a Muse Cell Cycle Assay Kit, 14 which displayed that Dex decreased the number of cells entering the $\mathrm{S}$ 15 phase (Figure 1C) and the expression of cell cycle control genes (P53, P21,

Dex upregulated the mRNA expression of DANCR 
1 Dex with three different concentrations $\left(1 \times 10^{-6}, 1 \times 10^{-7}, 1 \times 10^{-8} \mathrm{M}\right)$ was

2 added to the culture medium of hASCs individually. QPCR results showed

3 that Dex treatment increased the expression of DANCR, and was most

4 obvious on the third day. Additionally, Dex at concentrations of $10^{-6}$ and

$5 \quad 10^{-7} \mathrm{M}$ promoted DANCR expression more strongly (Figure 2).

6 DANCR inhibited cell proliferation and migration

7 We then investigated the role of DANCR in regulating cell proliferation

8 and migration characteristics, which are very important for the repair

9 effects of hASCs. We used lentivirus to overexpress DANCR in hASCs.

QPCR results showed a more than 15-fold increase of DANCR expression in the overexpression group (Figure 3A).

Overexpression of DANCR reduced hASCs proliferation ability (Figure 3B) from the second day of culture. Consistently, DANCR overexpression decreased mRNA expression of c-Jun and c-Fos (Figure 3C). An assessment of the percentage of cells in the G0/G1, S, and G2/M phase indicated a significantly lower cell number in the $\mathrm{S}$ phase and a higher one in G0/G1 phase in DANCR overexpressed cells (Figure 3D). The mRNA level of P53, P21, and CHEK1, which play crucial roles in the cell cycle control, was significantly increased in the DANCR overexpression group (Figure 3E). The migration results showed that DANCR-overexpressed hASCs migrated slower than that of the control group (Figure 3F), and a significant reduction of the migration-related genes, $C X C R 4$, and $C X C R 7$ 
3 Knockdown of DANCR reversed the inhibition of hASC proliferation

expression (Figure 3G).

\section{and migration induced by Dex}

We consequently investigated whether DANCR was involved in the decreased proliferation and migration of hASCs in response to Dex. We observed the effect of Dex on proliferation in hASCs transfected with shDANCR. The results of CCK- 8 and cell cycle assays indicated that knockdown of DANCR markedly reversed the reduction in hASC proliferation induced by Dex at $10^{-7} \mathrm{~mol} / \mathrm{l}$ concentration (Figure 4B, D). Cell migration experiments also reflected the reversing effects of DANCR on decreasing migration ability induced by Dex (Figure 4E). Similar results were further confirmed by the qRT-PCR results of proliferation and migration-related genes (Figure 4C, F).

\section{Inflammatory cytokines decreased the expression of DANCR}

To investigate whether DANCR was affected under inflammatory environment, six proinflammatory factors (TNF- $\alpha$, IL- $1 \beta$, IFN- $\gamma$, IL-6, IL8, and MCP-1) and two anti-inflammatory factors (IL-13 and IL-10) were added to the hASC culture media at $10 \mathrm{ng} / \mathrm{ml}$ and $50 \mathrm{ng} / \mathrm{ml}$ individually and the expression of DANCR was assessed by qRT-PCR analysis. TNF$\alpha$, IL-1 $\beta$, and IFN- $\gamma$ could significantly decrease the expression of DANCR 
1 in hASCs (Figure 5 A-C). And, IL-6, IL-8, IL-10, IL-13, and MCP-1 had

2 no significant effects on DANCR expression (Figure 5 D-H).

3 Overexpression of DANCR alleviated the promotion of hASC 4 proliferation and migration induced by TNF-a

5 To clarify whether DANCR was involved in the signal pathway of TNF- $\alpha$

6 regulating the cell characteristics of hASCs, we overexpressed DANCR in

7 hASCs and then added TNF- $\alpha$ stimulation in vitro to detect the changes in

8 cell proliferation and migration characteristics. The results showed that the 9 promotion effects of TNF- $\alpha$ on hASC proliferation and migration were significantly alleviated when DANCR was overexpressed. As shown by the CCK-8 assay, overexpression of DANCR partially reduced cell proliferation ability and related gene expression induced by TNF- $\alpha$ stimulation (Figure 6A, B). Cell cycle analysis results showed decreased cell numbers in the S phase and lower cell cycle control gene expression in DANCR overexpression group compared with TNF- $\alpha$ alone treated group (Figure 6C, D). Similarly, TNF- $\alpha$ could promote cell migration, but after overexpressing DANCR ( $\mathrm{pCDH}-\mathrm{DANCR}+\mathrm{TNF}-\alpha$ ), the migration ability of hASC was reduced, which was not statistically different from the control group (pCDH-GFP group) by 24h (Figure 6E). Besides, the expression of chemokine receptors CXCR4 and CXCR7 associated with cell migration was reduced (Figure 6F).

\section{DANCR was involved in the NF- $\mathrm{NB}$ signaling pathway}


1 Nuclear factor-kB (NF-kB) and PI3K-AKT-mTOR are two critical

2 signaling pathways that regulate the expression of some genes essential for

3 cell proliferation, apoptosis, and migration under inflammation conditions.

4 The results showed that TNF- $\alpha$ could activate NF- $\kappa$ B by inducing IKK $\alpha / \beta$

5 phosphorylation and $\mathrm{I} \kappa \mathrm{B} \alpha$ degradation as early as 5 minutes in hASC

6 (Figure 7A, B). Meanwhile, TNF- $\alpha$ significantly activated mTOR target

7 proteins p70S6K1 and AKT phosphorylation in hASCs within 5 minutes

8 and lasted 24 hours (Figure 7A, B). The NF-אB inhibitor BAY-117082

9 (BAY) and Dex were shown to inhibit the activation of phospho-IKK $\alpha / \beta$

10 induced by TNF- $\alpha$ in hASCs (Figure 7C). The mTOR inhibitors

11 Rapamycin (Rapa) and PP242 were shown to inhibit the TNF- $\alpha$-induced

12 activation of p70S6K (Figure 7D). To clarify the signal pathway that

DANCR played, the expression of DANCR was then assayed after TNF- $\alpha$ treatment in the presence or absence of inhibitors of the NF- $\mathrm{kB}$ and mTOR pathways. The NF- $\kappa \mathrm{B}$ inhibitors BAY and Dex could promote the expression of DANCR and significantly antagonize the downregulation of

DANCR induced by TNF- $\alpha$ in hASCs, while no significant difference was observed on the expression of DANCR after Rapa or PP242 treatment (Figure 7E). These results indicated that DANCR was involved in the NF$\kappa \mathrm{B}$ pathway in hASCs exposed to the inflammatory conditions.

\section{Discussion}

22 The ability to undergo self-renewal, migration, multi-differentiation, and 
1 immunomodulation is the most remarkable characteristic of hASCs, which

2 makes them particularly suited for various diseases, especially

3 inflammatory and immune-related disorders ${ }^{13-15}$.

4 Steroid-based anti-inflammatory therapy is often used to reduce severe

5 inflammatory responses in clinical practice. It is foreseen that hASCs may

6 be used for clinical purposes in combination with immunosuppressive

7 drugs. The molecular targets for Dex, which participate in the regulation of

8 biological characteristics of hASCs are important for potential clinical use

9 of hASCs in combination with immunosuppressant. In the present study, we investigated lncRNA DANCR expression profiles and their effects on cell proliferation and migration in response to Dex treatment in hASCs. We also showed that DANCR was involved in the inflammatory responses through the NF- $\kappa B$ pathway. These results help us further comprehend the molecular mechanism of cell biological characteristics of stem cells in the presence of immunosuppressive drugs in an inflammatory environment, and provide novel therapeutic targets for the application of hASCs.

DANCR is expressed and plays important roles in a variety of stem cells. Accordingly, we systemically identified the role of DANCR in hASCs for the first time and verified that overexpressing DANCR could decrease hASC proliferation ability. The results were consistent with another study, which showed that down-regulated DANCR promoted proliferation of PDLSCs $^{16}$. However, DANCR is a positive regulator of proliferation in 
1 most cancer cells, such as in nasopharyngeal carcinoma cells ${ }^{17}$ and glioma

2 cells ${ }^{18}$. These differences may be due to the different signaling pathways

3 of DANCR in different types of cells. DANCR knockdown inhibited EZH-

4 2-mediated epigenetic silencing of p21 promoter and increased p21

5 expression in non-small-cell lung cancer cells ${ }^{19}$. While in PDLSCs, the

6 effects of DANCR on cell proliferation is related to the activation of

7 canonical WNT pathway ${ }^{16}$. In our study, DANCR overexpression

8 decreased c-Jun and c-Fos gene expression and increased cell cycle-related

9 gene, p21, CHEK1, and p53 expression, while there was no difference in

10 EZH2 expression (unpublished data). Cell migration ability is another

11 important basis for stem cells to play a repair role. DANCR-overexpressed

12 hASC migrated slower than the control group. Therefore, the increased

13 expression of DANCR is not good for the reparability of hASCs.

14 In the present study, we found that Dex increased the expression of

15 DANCR, which suggested that the combined application of Dex and hASCs may not be conducive to its biological function of proliferation and

17 migration. The results of a clinical trial confirmed this. In the clinical trial using combined therapy with Prochymal, the first approved MSC drug in

Canada and New Zealand, along with steroids in graft-versus-host disease patients, there was no significant clinical improvement ${ }^{20}$. Our results showed that knockdown of DANCR reversed the inhibition of hASC proliferation and migration induced by Dex. These results provided a 
1 potential target for the combined application of hASC and Dex to improve

2 the repair effects.

3 TNF- $\alpha$ has been shown to have a pivotal role in the pathogenesis and

4 development of some autoimmune diseases, such as rheumatoid arthritis.

5 TNF- $\alpha$ is a major contributor to tissue inflammation, inducing a

6 proinflammatory cytokine cascade involving IL-1, IL-6, and IFN- $\gamma$, as well

7 as several proinflammatory chemokines ${ }^{[21]}$. In autoimmune disease, anti-

8 TNF- $\alpha$ antibodies control inflammation in most patients. Some studies

9 have demonstrated that Dex suppresses the production of endogenous

TNF- $\alpha$. We found that TNF- $\alpha$, IL- $1 \beta$, and IFN- $\gamma$ could decrease DANCR expression level, while IL-10 and IL-13, the anti-inflammatory factors had no effects on DANCR expression.

More importantly, overexpression of DANCR alleviated the promotion of hASC proliferation and migration induced by TNF- $\alpha$. In vitro studies suggest that inflammatory signals such as TNF-a or IL-1 $\beta$ could trigger the proliferation of NSPCs through NF-kB and JNK signaling pathways, respectively ${ }^{22,23}$. We also found that TNF- $\alpha$ significantly promoted hASC proliferation and migration, demonstrating that acute inflammation was positively affecting hASC characteristics. Therefore, in the early stage of inflammation, TNF- $\alpha$ could reduce the expression of DANCR, thereby promoting the proliferation and migration of hASCs.

Mechanically, we found that TNF- $\alpha$ could activate NF- $\kappa B$ and mTOR 
1 signal pathways in hASC by inducing IKK $\alpha / \beta$ and AKT phosphorylation,

2 respectively. Dex was shown to inhibit TNF- $\alpha$-induced activation of

3 phospho-IKK $\alpha / \beta$ in hASC. Besides, the NF- $\kappa \mathrm{B}$ inhibitors BAY and Dex

4 could significantly antagonize the downregulation of DANCR induced by

5 TNF- $\alpha$ in hASC, implying the role of DANCR in the NF- $\kappa B$ pathway.

6 However, the precise molecular mechanism by which the DANCR acts on

7 cell proliferation and migration-related genes is not fully understood.

8 In summary, we identified a lncRNA, DANCR, involved in Dex and

9 inflammation-affected hASC proliferation and migration, thus suggesting

10 that concurrent application of hASC with steroids should be avoided in

11 clinical settings. DANCR may serve as a promising target to regulate stem

12 cell characteristics under an inflamed microenvironment. These findings

13 further enrich our understanding of the functional versatility of lncRNAs

14 in the crosstalk of inflammation conditions and stem cells.

\section{Conclusion}

Dex increased the expression of lncRNA DANCR. Knockdown of

DANCR not only promoted hASC proliferation and migration but also reversed the inhibition induced by Dex. Acute inflammation factor TNF- $\alpha$ positively affected hASC proliferation and migration by decreasing DANCR expression. DANCR is involved in the NF- $\kappa B$ signaling pathway. Inhibition of the NF- $\mathrm{kB}$-DANCR pathway may have benefits in enhancing 
3 Abbreviations

4

5 Differentiation antagonizing non-protein coding RNA; hASCs: human

hASC proliferation and migration in early inflammation conditions.

MSCs: Mesenchymal Stem Cells; Dex: Dexamethasone; DANCR: Adipose Derived Stem Cells; TNF- $\alpha$ : Tumor Necrosis Factor alpha; qRTPCR: Real-time Quantitative Reverse Transcription-Polymerase Chain

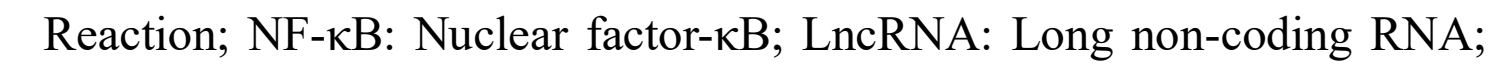
SVF: Stromal Vascular Fraction; PBS: Phosphate Buffer Saline; MSCM: Mesenchymal Stem Cell Medium; FBS: Fetal Bovine Serum; IL-1 $\beta$ : Interleukin 1 beta; IFN- $\gamma$ : Interferon $\gamma$; IL-6: Interleukin 6; IL-8: Interleukin 8; MCP-1: Monocyte Chemoattractant Protein-1; IL-13: Interleukin 13; IL-10: Interleukin 10; CCK-8: Cell Counting Kit-8; GFP: Green Fluorescence Protein; WB: Western blot; IKK- $\beta$ : inhibitor of nuclear

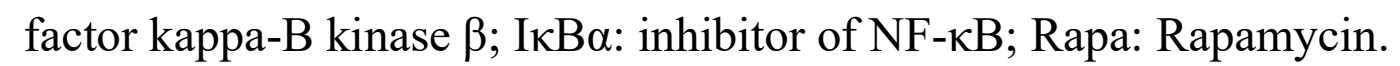

\section{Authors' contributions}

RY performed the research, data analysis. PD, ZGY and RC participated in the research and data collection. XL, and RX contributed to the design of the study and manuscript writing and revised. All authors read and approved the final manuscript. 


\section{$2 \quad$ Funding}

3 This study was supported by the National Natural Science Foundation of

4 China $(81873666,81871575)$, the CAMS Innovation Fund for Medical

5 Sciences (CIFMS, grant no. 2016-I2M-1-017), and Program for Union

6 Scholars and Innovative Research Team in Peking Union Medical College,

7 and Non-profit Central Research Institute Fund of Chinese Academy of

8 Medical Sciences (2018PT32015).

9

10

\section{Availability of data and materials}

The datasets during the current study are available from the corresponding authors on a reasonable request.

\section{Ethics approval and consent to participate}

The research was reviewed and approved by the Ethics Committee of the Plastic Surgery Hospital, Chinese Academy of Medical Sciences, Peking Union Medical College.

\section{Competing interests}

The authors declare that they have no competing interests. 
2 1. Gimble JM, Katz AJ, Bunnell BA. Adipose-derived stem cells for 3 regenerative medicine. Circ Res 2007; 100(9):1249-60.

4

\section{References}

2. Baer PC, Geiger H. Adipose-derived mesenchymal stromal/stem cells: tissue localization, characterization, and heterogeneity. Stem Cells Int $2012 ; 2012: 812693$.

3. Wang H, Pang B, Li Y, Zhu D, Pang T, Liu Y.Dexamethasone has variable effects on mesenchymal stromal cells. Cytotherapy. 2012; 14(4):423-30.

4. Xiao Y, Peperzak V, van Rijn L, Borst J, de Bruijn JD. Dexamethasone treatment during the expansion phase maintains stemness of bone marrow mesenchymal stem cells.J Tissue Eng Regen Med. 2010; 4(5):374-86.

5. Ghosal S, Das S, Chakrabarti J. Long noncoding RNAs: new players in the molecular mechanism for maintenance and differentiation of pluripotent stem cells. Stem Cells Dev. 2013; 22(16):2240-53.

6. Nam JW, Choi SW, You BH. Incredible RNA: Dual Functions of Coding and Noncoding. Mol Cells. 2016; 39(5):367-74.

7. $\mathrm{Hu}$ S, Shan G. LncRNAs in Stem Cells. Stem Cells Int. 2016; 2016:2681925.

8. Rapicavoli NA, Qu K, Zhang J, Mikhail M, Laberge RM, Chang HY. A mammalian pseudogene lncRNA at the interface of inflammation and 
anti-inflammatory therapeutics. Elife 2013; 2:e00762.

2 9. Carpenter S, Aiello D, Atianand MK, Ricci EP, Gandhi P, Hall LL, et al.

3 A long noncoding RNA mediates both activation and repression of

$4 \quad$ immune response genes. Science 2013; 341(6147):789-92.

5 10. Kretz M, Webster DE, Flockhart RJ, Lee CS, Zehnder A, Lopez-Pajares

$6 \mathrm{~V}$, et al. Suppression of progenitor differentiation requires the long 7 noncoding RNAANCR. Genes Dev. 2012; 26(4):338-43.

8 11. Jia Q, Chen X, Jiang W, Wang W, Guo B, Ni L.The Regulatory Effects 9 of Long Noncoding RNA-ANCR on Dental Tissue-Derived Stem Cells. Stem Cells Int. 2016;2016:3146805.

12. Li J, Yang Y, Fan J, Xu H, Fan L, Li H, et al. Long noncoding RNA ANCR inhibits the differentiation of mesenchymal stem cells toward definitive endoderm by facilitating the association of PTBP1 with ID2. Cell Death Dis. 2019; 10(7):492.

13. Andreeva E, Andrianova I, Bobyleva P, Gornostaeva A, Ezdakova M, Golikova E, et al. Adipose tissue-derived stromal cells retain immunosuppressive and angiogenic activity after coculture with cord blood hematopoietic precursors. Eur J Cell Biol. 2020;99(2-3):151069. 14. Choi EW, Lee HW, Shin IS, Park JH, Yun TW, Youn HY, et al. Comparative Efficacies of Long-Term Serial Transplantation of Syngeneic, Allogeneic, Xenogeneic, or CTLA4Ig-Overproducing Xenogeneic Adipose Tissue-Derived Mesenchymal Stem Cells on 
Murine Systemic Lupus Erythematosus. Cell Transplant.

2 2016;25(6):1193-206.

15. Lopez-Santalla M, Mancheño-Corvo P, Menta R, Lopez-Belmonte J, DelaRosa O, Bueren JA, et al. Human Adipose-Derived Mesenchymal Stem Cells Modulate Experimental Autoimmune Arthritis by Modifying Early Adaptive T Cell Responses. Stem Cells. 2015; 33(12):3493-503.

16. Jia Q, Jiang W, Ni L. Down-regulated non-coding RNA (lncRNAANCR) promotes osteogenic differentiation of periodontal ligament stem cells. Arch Oral Biol. 2015; 60(2):234-41.

17. Hao Y, Zhao H, Jin X, He P, Zhang J, Dong Q,et al. Long non-coding RNA DANCR promotes nasopharyngeal carcinoma cell proliferation and migration. Mol Med Rep. 2019; 19(4):2883-2889.

18. Feng L, Lin $\mathrm{T}$, Che $\mathrm{H}$, Wang $\mathrm{X}$. Long noncoding RNA DANCR knockdown inhibits proliferation, migration and invasion of glioma by regulating miR-135a-5p/BMI1. Cancer Cell Int. 2020; 20:53.

19. Guo L, Gu J, Hou S, Liu D, Zhou M, Hua T, et al. Long non-coding RNA DANCR promotes the progression of non-small-cell lung cancer by inhibiting p21 expression. Onco Targets Ther. 2018; 12:135-146.

20. X Chen, Y Gan, W Li, J Su, Y Zhang, Y Huang, et al. The interaction between mesenchymal stem cells and steroids during inflammation. Cell Death Dis. 2014; 5(1): e1009.

21. Erlandsson A, Lin $\mathrm{CH}, \mathrm{Yu}$ F, Morshead CM. Immunosuppression 
1 promotes endogenous neural stem and progenitor cell migration and

2 tissue regeneration after ischemic injury. Exp Neurol. 2011; 230(1):48357.

4 22. Widera D, Mikenberg I, Elvers M, Kaltschmidt C, Kaltschmidt B. 5 Tumor necrosis factor alpha triggers proliferation of adult neural stem 6 cells via IKK/NF-kappaB signaling. BMC Neurosci. 2006; 7:64.

7 23. Wang $\mathrm{X}, \mathrm{Fu} \mathrm{S}$, Wang $\mathrm{Y}, \mathrm{Yu} \mathrm{P}, \mathrm{Hu} \mathrm{J}, \mathrm{Gu}$ W, et al. Interleukin-1beta 8 mediates proliferation and differentiation of multipotent neural 9 precursor cells through the activation of SAPK/JNK pathway. Mol Cell Neurosci. 2007; 36(3):343-54.

Figure legends hASC proliferation under different doses of Dex tested by the CCK8 method. (B) qRT-PCR analysis of genes associated with proliferation (cJun and c-Fos). (C) Cell cycle distribution of hASC under different doses 22 of Dex analyzed with a Muse Cell Analyzer. (D) qRT-PCR analysis of 
1 genes associated with the cell cycle (P53, P21, and CHEK1). (E) hASC

2 migration under different doses of Dex tested by scratching test. Percentage

3 of scratch healing was quantified as (scratch area ${ }_{0 h}$-scratch area $\left.{ }_{x h}\right) /$ scratch

4 area $_{0 \mathrm{~h}} * 100 \%$. (F) qRT-PCR analysis of genes associated with migration

5 (CXCR4 and CXCR7). Results are presented as the mean $\pm \mathrm{SD}(\mathrm{n}=3$,

$\left.6 \quad * \mathrm{p}<0.05,{ }^{*} \mathrm{p}<0.01, * * * \mathrm{p}<0.001\right)$.

7 Figure 2 DANCR expression was increased by Dexamethasone. qRT-PCR 8 analysis of DANCR expression under different doses of Dex (normalized 9 by GAPDH; relative to shScramble groups). Results are presented as the mean \pm SD $(\mathrm{n}=3, * \mathrm{p}<0.05, * * \mathrm{p}<0.01, * * * \mathrm{p}<0.001)$. Abbreviations: Dex, 11 dexamethasone.

Figure 3 LncRNA DANCR inhibited cell proliferation and migration. (A) qRT-PCR analysis of DANCR expression after DANCR overexpression. (B) hASC proliferation after DANCR overexpression tested by the CCK8 method. (C) qRT-PCR analysis of c-Jun and c-Fos gene expression after DANCR overexpression. (D) Cell cycle distribution of hASC after DANCR overexpression. (E) qRT-PCR analysis of P53, P21, and CHEK1 gene expression after DANCR overexpression. (F) hASC migration after DANCR overexpression tested by scratch test. Percentage of scratch healing was quantified as (scratch area ${ }_{0 \mathrm{~h}}$-scratch area $\left.{ }_{\mathrm{xh}}\right) /$ scratch area $\mathrm{oh} *$ 100\%. (G) qRT-PCR analysis $C X C R 4$ and $C X C R 7$ gene expression after DANCR overexpression. Results are presented as the mean \pm SD ( $n=3$, 
2 Figure 4 Knockdown of DANCR reversed the inhibition of hASC

$\left.{ }^{*} \mathrm{p}<0.05, * * \mathrm{p}<0.01, * * * \mathrm{p}<0.001\right)$. proliferation and migration induced by Dex. (A) Relative mRNA expression of DANCR in shScramble, shDANCR1, and shDANCR2 groups with or without $1 \times 10^{-7} \mathrm{~mol} / 1$ Dex treatment for 72 hours (Normalized by GAPDH; relative to shScramble group). (B) hASC proliferation in shScramble, shDANCR1, and shDANCR2 groups treated with or without $1 \times 10^{-7} \mathrm{~mol} / 1$ Dex tested by CCK 8 method. (C) Relative mRNA expression of $c$-Fos and $c$-Jun measured by qRT-PCR (Normalized by $G A P D H$; relative to shScramble group). (D) Cell cycle distribution of hASC in shScramble, shDANCR1, and shDANCR2 groups with or without $1 \times 10^{-7} \mathrm{~mol} / 1$ Dex treatment analyzed with a Muse Cell Analyzer. (E) Images and quantitative data from the scratch migration assay in shScramble, shDANCR1, and shDANCR2 groups with or without $1 \times 10^{-7}$ mol/1 Dex treatment. Percentage of scratch healing was quantified as (scratch area ${ }_{0 h}$-scratch area $\left.x_{\mathrm{xh}}\right) /$ scratch area $_{0 \mathrm{~h}} * 100 \%$. (F) Relative mRNA expression of chemokine receptors $C X C R 4$ and $C X C R 7$ measured by qRTPCR (Normalized by GAPDH; relative to shScramble group). Results are presented as the mean \pm SD $(n=3, * p<0.05, * * p<0.01, * * * p<0.001)$. Abbreviations: Dex, dexamethasone.

Figure 5 Inflammatory cytokines decreased DANCR expression. qRT-PCR analysis of DANCR expression in hASC treated with (A) $10,50 \mathrm{ng} / \mathrm{ml}$ 
$1 \mathrm{TNF}-\alpha$; (B) 10, $50 \mathrm{ng} / \mathrm{ml} \mathrm{IL-1 \beta ;} \mathrm{(C)} \mathrm{10,} 40 \mathrm{ng} / \mathrm{ml} \mathrm{IFN- \gamma ;} \mathrm{(D)} \mathrm{10,} 40 \mathrm{ng} / \mathrm{ml}$

2 IL-6; (E) 10, 40 ng/ml IL-8; (F) 10, 40 ng/ml IL-10; (G) 10, 40 ng/ml IL-

3 13; (H) 10, $40 \mathrm{ng} / \mathrm{ml} \mathrm{MCP-1} \mathrm{for} 24 \mathrm{~h}$ and $72 \mathrm{~h}$. (Normalized by GAPDH;

4 relative to control group). Results are presented as the mean $\pm \mathrm{SD}(\mathrm{n}=3$, $5 \quad * * \mathrm{p}<0.01, * * * \mathrm{p}<0.001)$.

6 Figure 6 Overexpression of DANCR alleviated the promotion of hASC 7 proliferation and migration induced by $10 \mathrm{ng} / \mathrm{ml}$ TNF- $\alpha$. (A) The 8 proliferation of hASC in pCDHGFP and pCDHDANCR groups treated 9 with $10 \mathrm{ng} / \mathrm{ml}$ TNF- $\alpha$. (B) Relative mRNA expression of $c-F o s$ and c-Jun genes measured by qRT-PCR (Normalized by GAPDH; relative to PCDHGFP group). (C) Cell cycle distribution of hASC in pCDHGFP and pCDHDANCR groups treated with 10ng/ml TNF- $\alpha$. (D) Relative mRNA expression of P53, P21, and CHEK1 genes measured by qRT-PCR (Normalized by GAPDH; relative to PCDHGFP group). (E) Images and quantitative data from the scratch migration assay in pCDHGFP and pCDHDANCR groups treated with 10ng/ml TNF- $\alpha$. Percentage of scratch healing was quantified as (scratch area ${ }_{0 \mathrm{~h}}$-scratch area $\left.\mathrm{xh}_{\mathrm{h}}\right) /$ scratch area ${ }_{0 \mathrm{~h}}$ * $100 \%$. (F) Relative mRNA expression of chemokine receptors $C X C R 4$ and CXCR7 measured by qRT-PCR (Normalized by GAPDH; relative to PCDHGFP group). Results are presented as the mean \pm SD $\left(n=3,{ }^{*}<<0.05\right.$, $* * \mathrm{p}<0.01)$

Figure 7 DANCR was involved in the NF- $\kappa$ B signaling pathway. (A) The 
1 effect of $10 \mathrm{ng} / \mathrm{ml}$ TNF- $\alpha$ on phosphorylated $\operatorname{IKK} \alpha / \beta, \quad$ I $\kappa \mathrm{B} \alpha$,

2 phosphorylated and pan p70s6 kinase, phosphorylated and pan AKT

3 protein expression was assayed by $\mathrm{WB}$ analysis. $\mathrm{B}$-actin was used as a

4 loading control. (B) Densitometry assay with Image J. (C) The NF-kB

5 inhibitors BAY and Dex inhibited TNF- $\alpha$-induced activation of phospho-

$6 \quad$ IKK $\alpha / \beta$. (D) The mTOR inhibitors Rapa and PP242 inhibited the TNF- $\alpha$

7 induced phosphorylation of p70s6 kinase. (E) DANCR expression was

8 affected by NF-kB inhibitors BAY and Dex, while Rapa and PP242 had no

9 effects. Results are presented as the mean $\pm \mathrm{SD}\left(\mathrm{n}=3,{ }^{*} \mathrm{p}<0.05, * * \mathrm{p}<0.01\right.$,

10 relative to control group; $\# \mathrm{p}<0.05$, relative to TNF- $\alpha$ group). Abbreviations:

11 BAY, BAY-117082; Dex, dexamethasone; Rapa, rapamycin. 


\section{Figures}
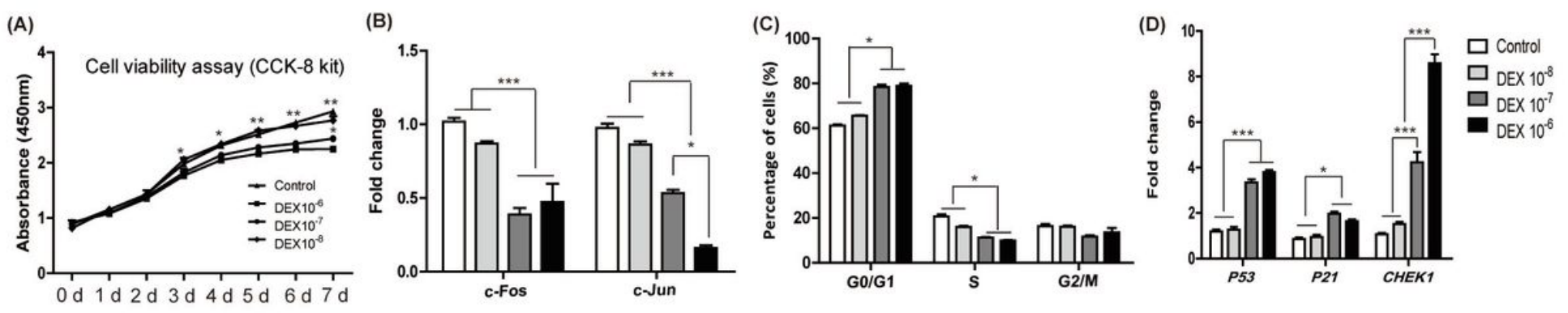

(E)
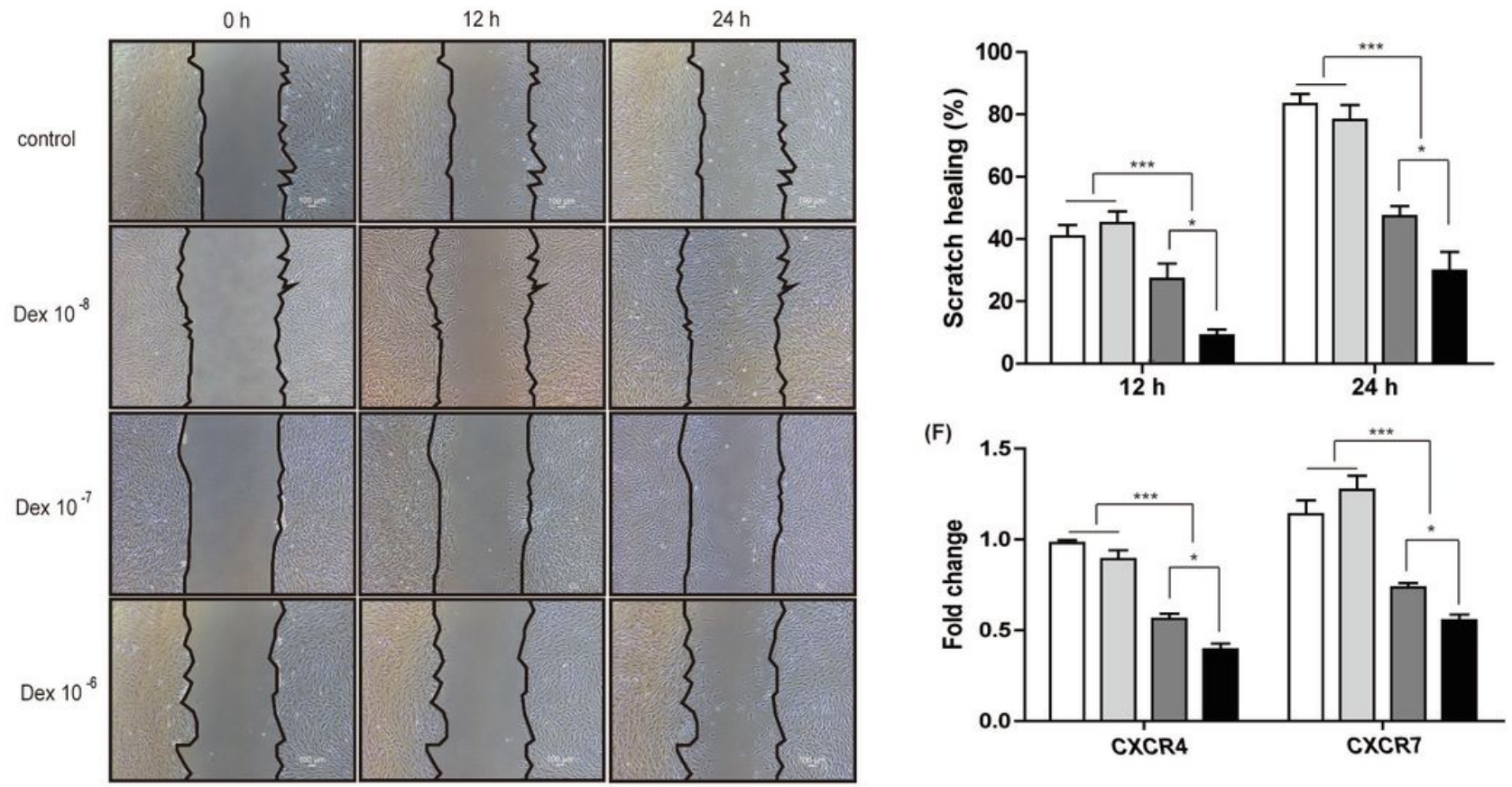

\section{Figure 1}

Dex decreased the proliferation and migration of hASC. (A) hASC proliferation under different doses of Dex tested by the CCK8 method. (B) qRT-PCR analysis of genes associated with proliferation (c- Jun and C-Fos). (C) Cell cycle distribution of hASC under different doses of Dex analyzed with a Muse Cell Analyzer. (D) qRT-PCR analysis of genes associated with the cell cycle (P53, P21, and 1 CHEK1). (E) hASC migration under different doses of Dex tested by scratching test. Percentage of scratch healing was quantified as (scratch area Oh-scratch area $\mathrm{xh}$ )/ scratch area $0 \mathrm{~h} * 100 \%$. (F) qRT-PCR analysis of genes associated with migration (CXCR4 and CXCR7). Results are presented as the mean $\pm S D(n=3, * p<0.05$, $\left.{ }^{* \star} p<0.01,{ }^{* \star *} p<0.001\right)$. 


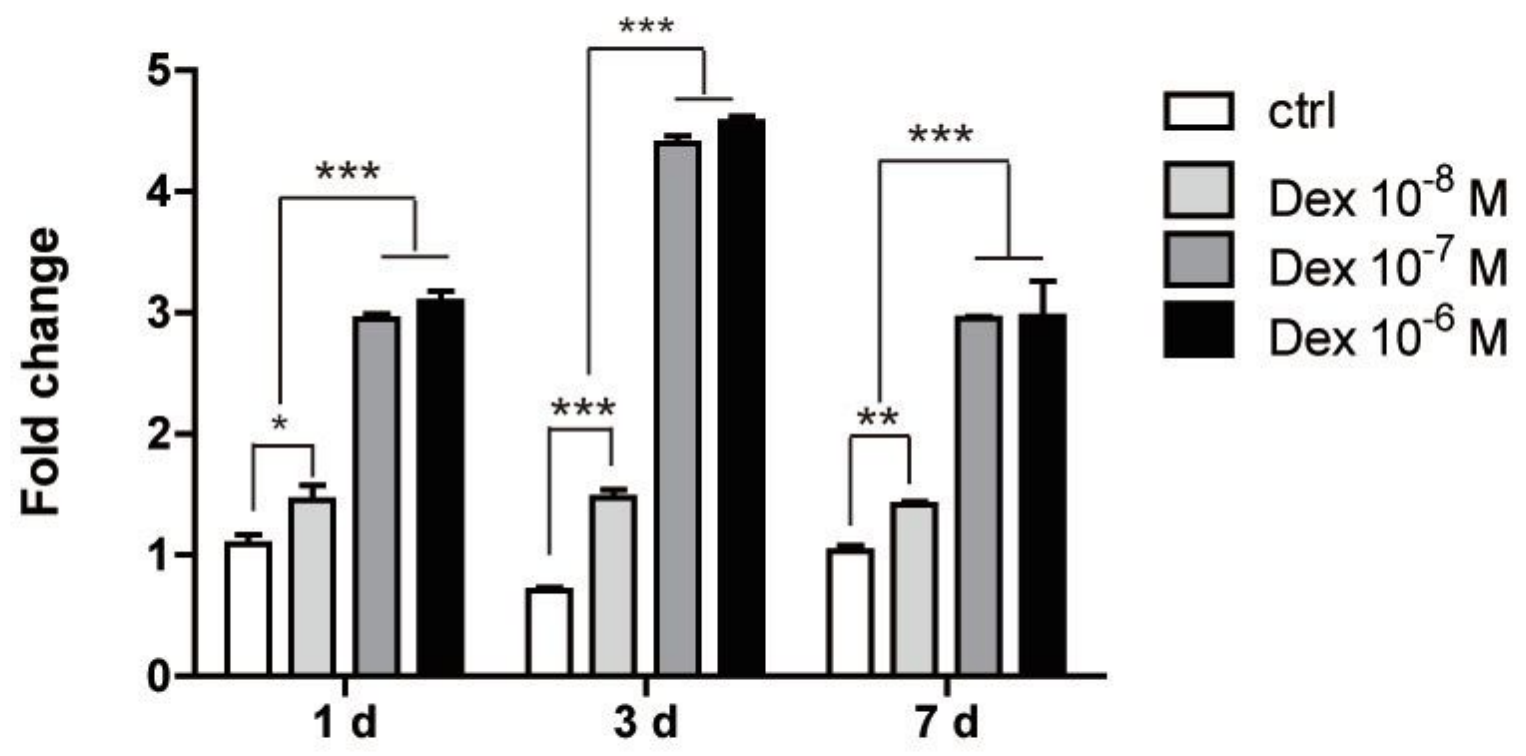

Figure 2

DANCR expression was increased by Dexamethasone. qRT-PCR analysis of DANCR expression under different doses of Dex (normalized by GAPDH; relative to shScramble groups). Results are presented as the mean $\pm S D\left(n=3,{ }^{*}<<0.05,{ }^{* \star} p<0.01,{ }^{* * *} p<0.001\right)$. Abbreviations: Dex, dexamethasone. 
(A)

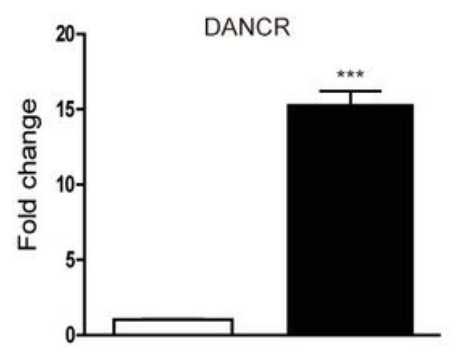

(D)

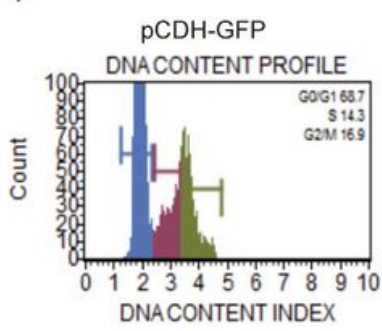

(B)

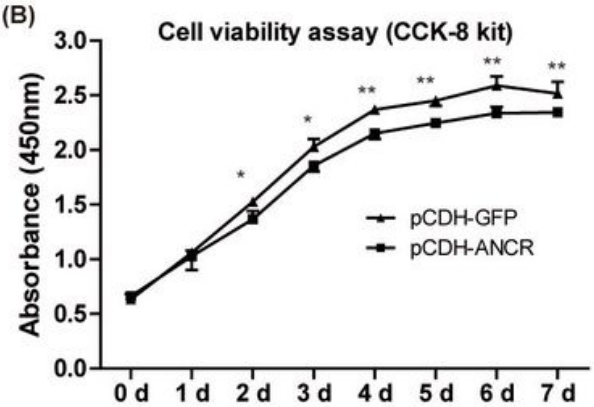

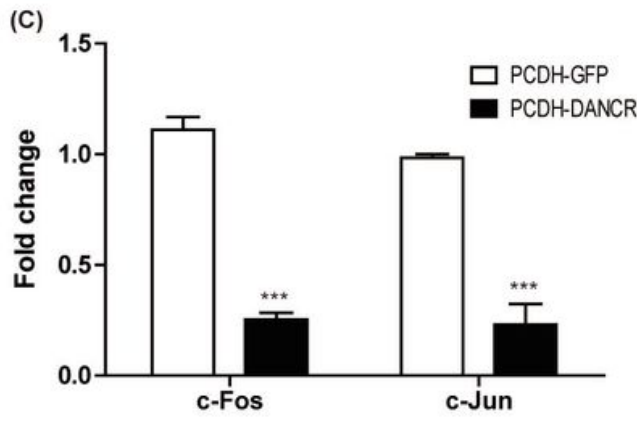

$(\mathrm{E})$
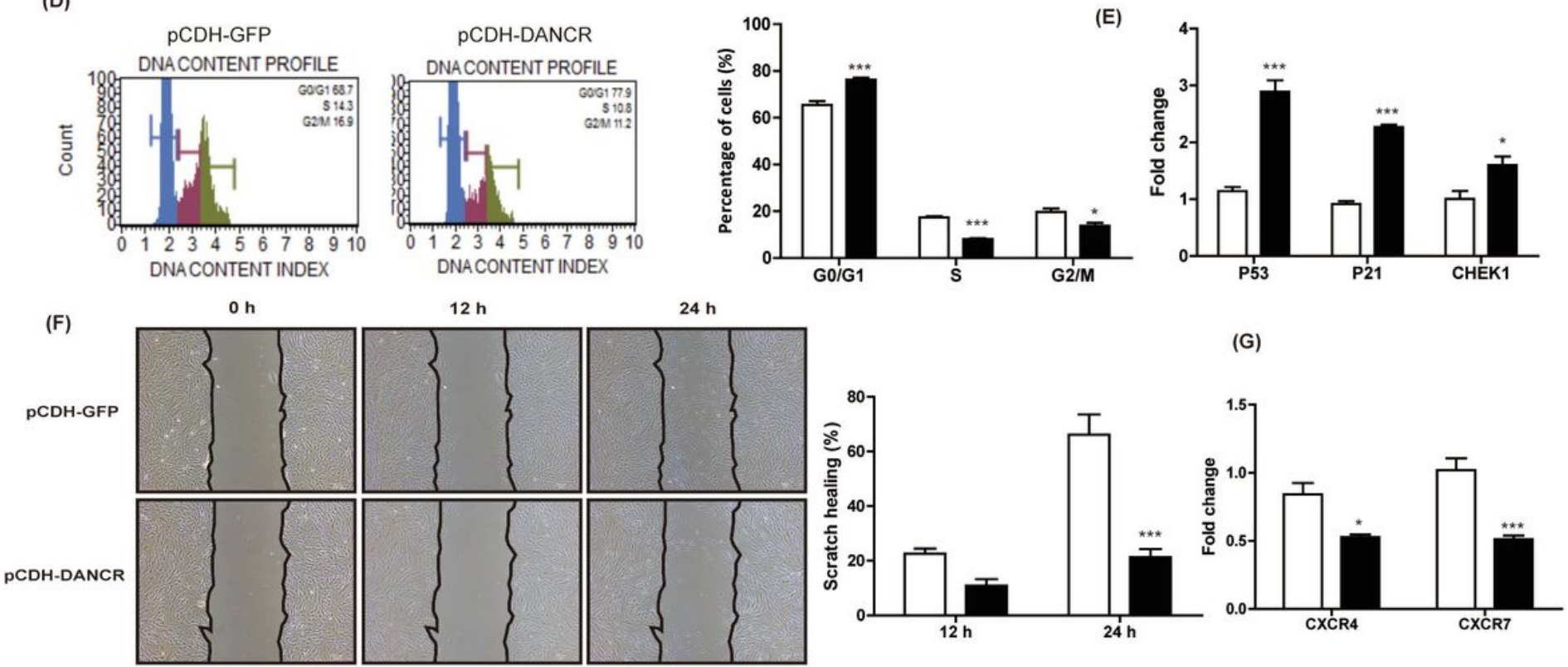

(G)

Figure 3

LncRNA DANCR inhibited cell proliferation and migration. (A) qRT-PCR analysis of DANCR expression after DANCR overexpression. (B) hASC proliferation after DANCR overexpression tested by the CCK8 method. (C) qRT-PCR analysis of c-Jun and c-Fos gene expression after DANCR overexpression. (D) Cell cycle distribution of hASC after DANCR overexpression. (E) qRT-PCR analysis of P53, P21, and CHEK1 gene expression after DANCR overexpression. (F) hASC migration after DANCR overexpression tested by scratch test. Percentage of scratch healing was quantified as (scratch area Oh-scratch area $\mathrm{xh}$ )/ scratch area $0 \mathrm{~h} * 100 \%$. (G) qRT-PCR analysis CXCR4 and CXCR7 gene expression after DANCR overexpression. Results are presented as the mean $\pm S D(n=3, * p<0.05, * \star p<0.01,1 * \star * p<0.001)$. 

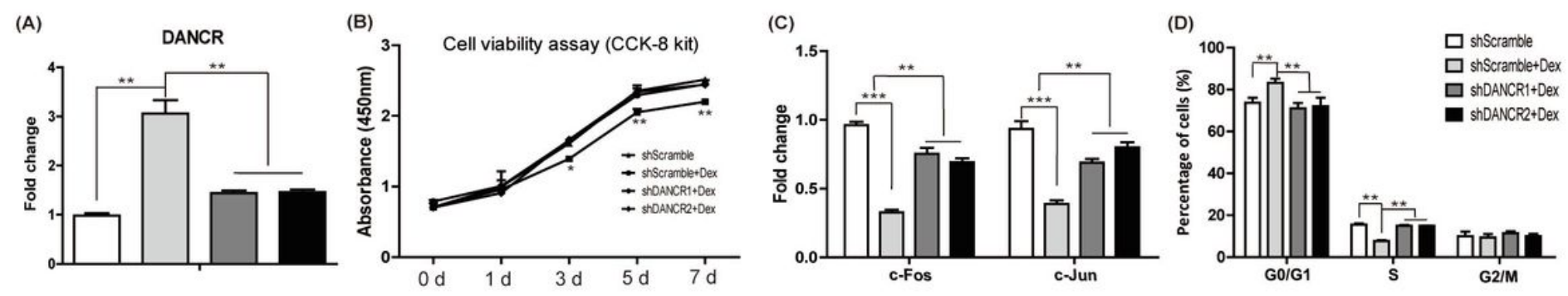

(E)
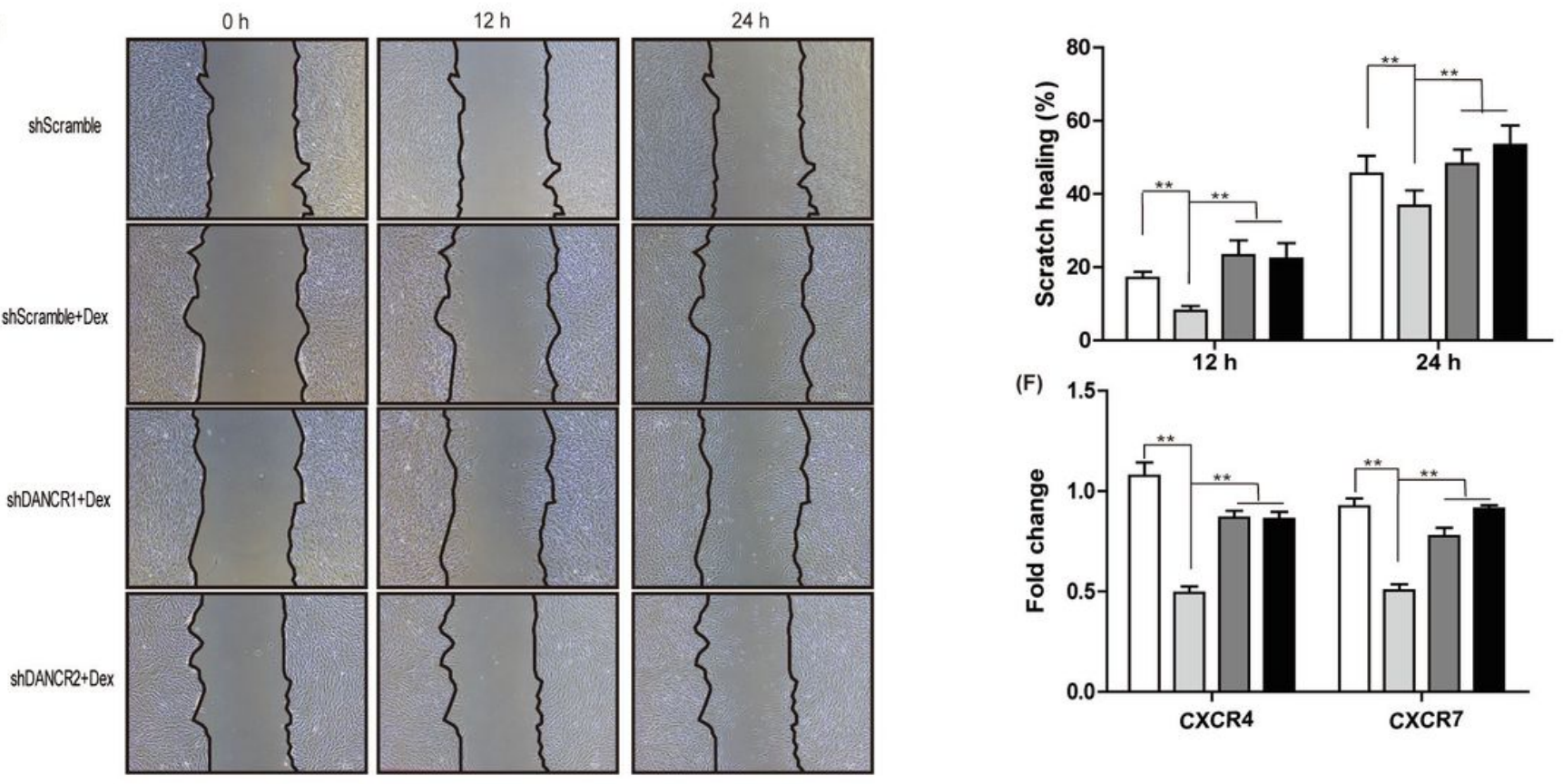

\section{Figure 4}

Knockdown of DANCR reversed the inhibition of hASC proliferation and migration induced by Dex. (A) Relative mRNA expression of DANCR in shScramble, shDANCR1, and shDANCR2 groups with or without 1×10-7 mol/I Dex treatment for 72 hours (Normalized by GAPDH; relative to shScramble group). (B) hASC proliferation in shScramble, shDANCR1, and shDANCR2 groups treated with or without $1 \times 10-7 \mathrm{~mol} / \mathrm{I}$ Dex tested by CCK8 method. (C) Relative mRNA expression of c-Fos and c-Jun measured by qRT-PCR (Normalized by GAPDH; relative to shScramble group). (D) Cell cycle distribution of hASC in shScramble, shDANCR1, and shDANCR2 groups with or without 1×10-7 mol/I Dex treatment analyzed with a Muse Cell Analyzer. (E) Images and quantitative data from the scratch migration assay in shScramble, shDANCR1, and shDANCR2 groups with or without $1 \times 10-7 \mathrm{~mol} / \mathrm{I}$ Dex treatment. Percentage of scratch healing was quantified as (scratch area 0h-scratch area $x h$ )/ scratch area $0 \mathrm{~h} * 100 \%$. (F) Relative mRNA expression of chemokine receptors CXCR4 and CXCR7 measured by qRT18 PCR (Normalized by GAPDH; relative to shScramble group). Results are presented as the mean $\pm S D\left(n=3,{ }^{*} p<0.05,{ }^{\star \star} p<0.01,{ }^{\star \star \star} p<0.001\right)$. Abbreviations: Dex, dexamethasone. 
(A)

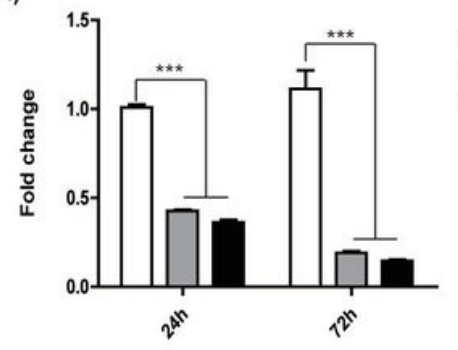

(D)

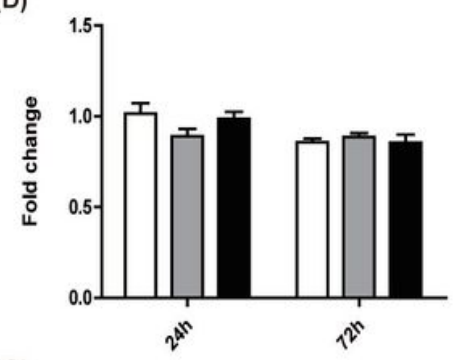

(G)

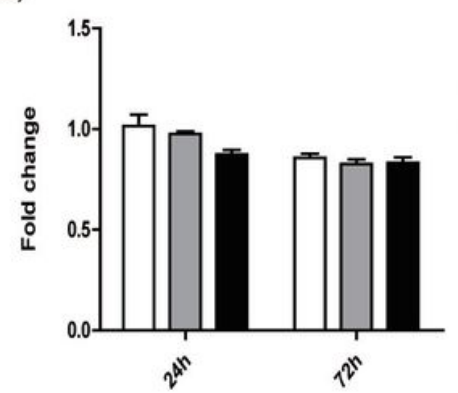

(B)
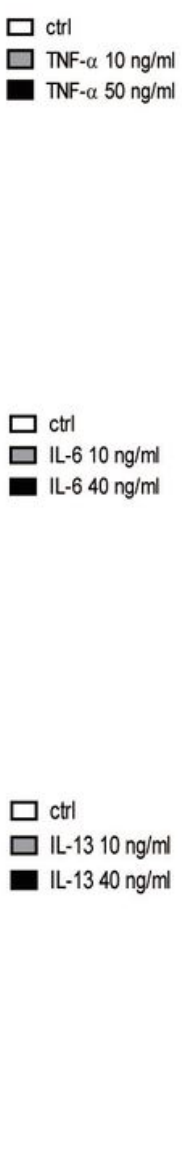

(E)
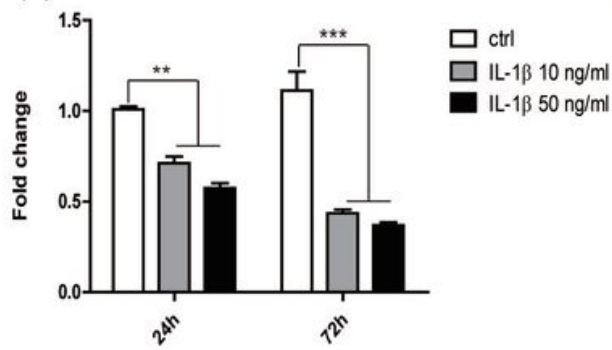

(C)

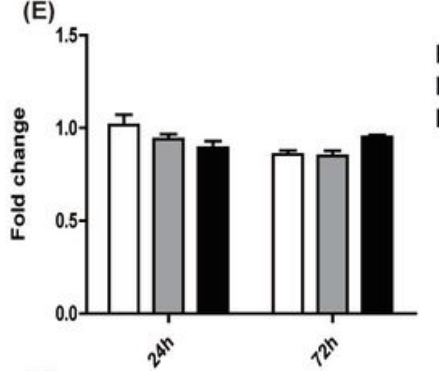

(H)

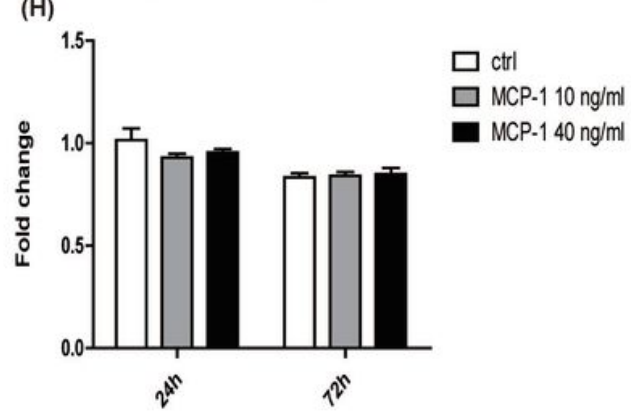

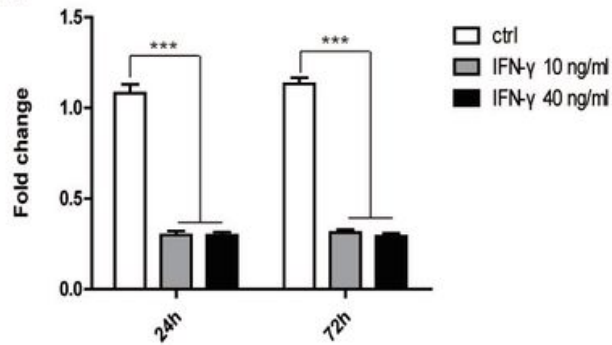

(F)

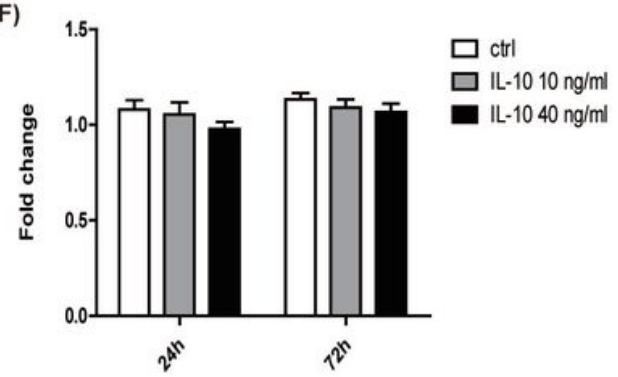

Figure 5

Inflammatory cytokines decreased DANCR expression. qRT-PCR analysis of DANCR expression in hASC treated with (A) 10, $50 \mathrm{ng} / \mathrm{ml}$ TNF-a; (B) 10, $50 \mathrm{ng} / \mathrm{ml} \mathrm{IL-1 \beta ;} \mathrm{(C)} \mathrm{10,} 40 \mathrm{ng} / \mathrm{ml} \mathrm{IFN-1} \mathrm{\gamma ;} \mathrm{(D)} \mathrm{10,} 40 \mathrm{ng} / \mathrm{ml}$ IL-6; (E) 10, $40 \mathrm{ng} / \mathrm{ml} \mathrm{IL-8;} \mathrm{(F)} \mathrm{10,} 40 \mathrm{ng} / \mathrm{ml} \mathrm{IL-10;} \mathrm{(G)} \mathrm{10,} 40 \mathrm{ng} / \mathrm{ml} \mathrm{IL-13;} \mathrm{(H)} \mathrm{10,} 40 \mathrm{ng} / \mathrm{ml} \mathrm{MCP-1}$ for 24h and 72h. (Normalized by GAPDH; relative to control group). Results are presented as the mean $\pm S D(n=3$, $\star * p<0.01, * \star * p<0.001)$. 

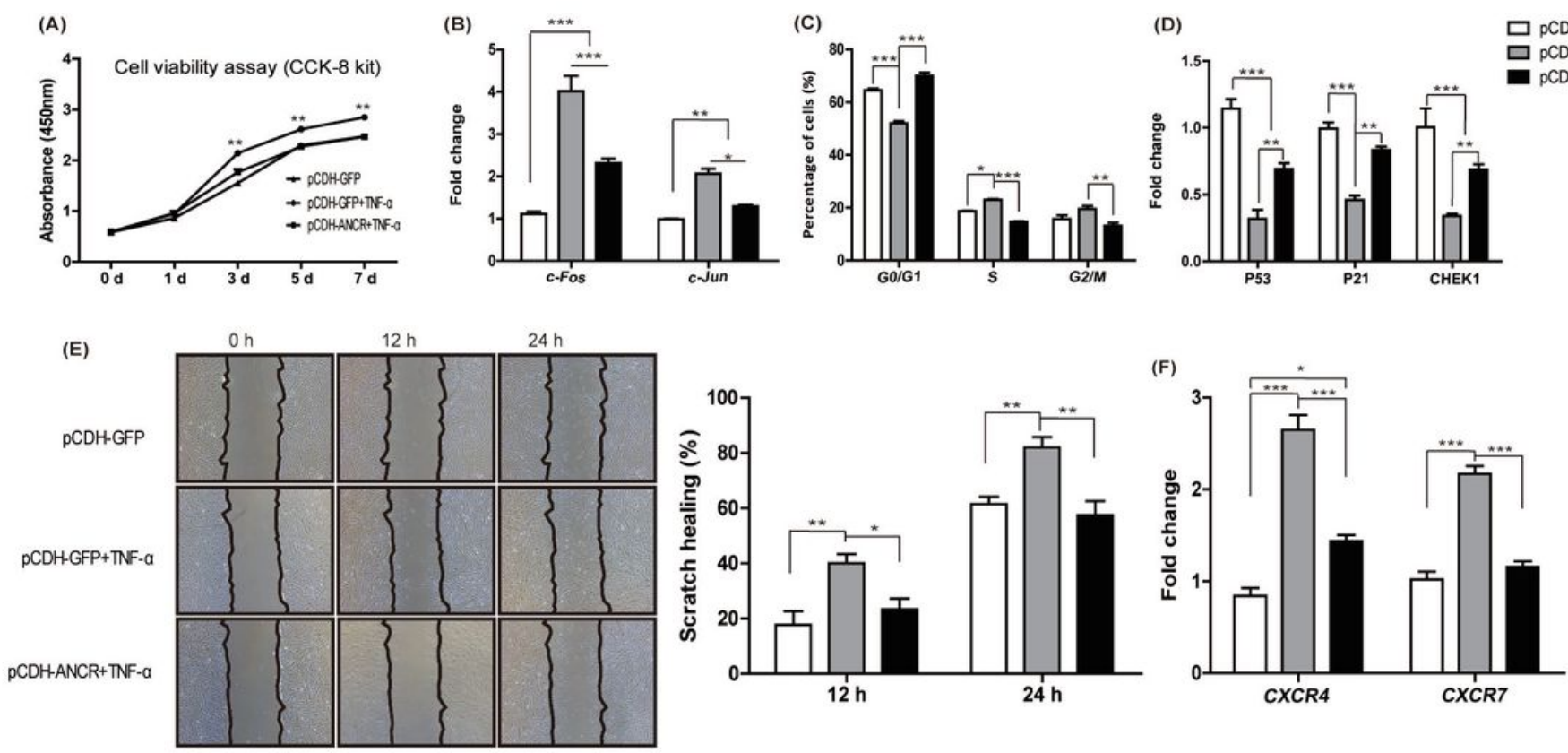

\section{Figure 6}

Overexpression of DANCR alleviated the promotion of hASC proliferation and migration induced by 10ng/ml TNF-a. (A) The proliferation of hASC in pCDHGFP and pCDHDANCR groups treated with 10ng/ml TNF-a. (B) Relative mRNA expression of c-Fos and c-Jun genes measured by qRT-PCR (Normalized by GAPDH; relative to PCDHGFP group). (C) Cell cycle distribution of hASC in pCDHGFP and pCDHDANCR groups treated with 10ng/ml TNF-a. (D) Relative mRNA expression of P53, P21, and CHEK1 genes measured by qRT-PCR (Normalized by GAPDH; relative to PCDHGFP group). (E) Images and quantitative data from the scratch migration assay in pCDHGFP and PCDHDANCR groups treated with $10 \mathrm{ng} / \mathrm{ml}$ TNF-a. Percentage of scratch healing was quantified as (scratch area Oh-scratch area $\mathrm{xh}$ )/ scratch area $0 \mathrm{~h} * 100 \%$. (F) Relative mRNA expression of chemokine receptors CXCR4 and CXCR7 measured by qRT-PCR (Normalized by GAPDH; relative to PCDHGFP group). Results are presented as the mean $\pm S D\left(n=3,{ }^{*}<<0.05,{ }^{*} p<0.01\right)$. 
(A)

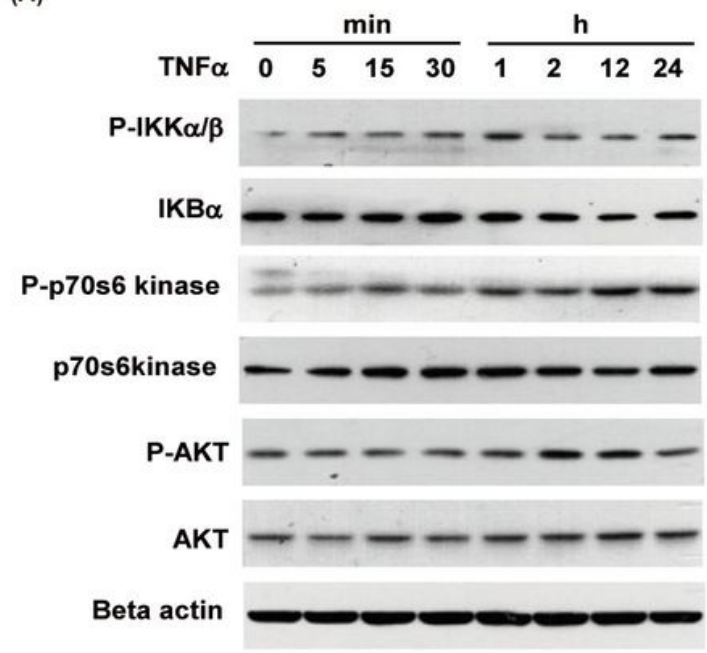

(C)
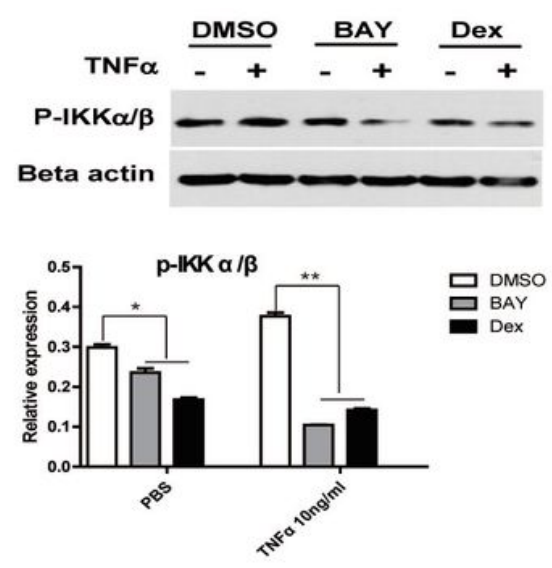

(B)
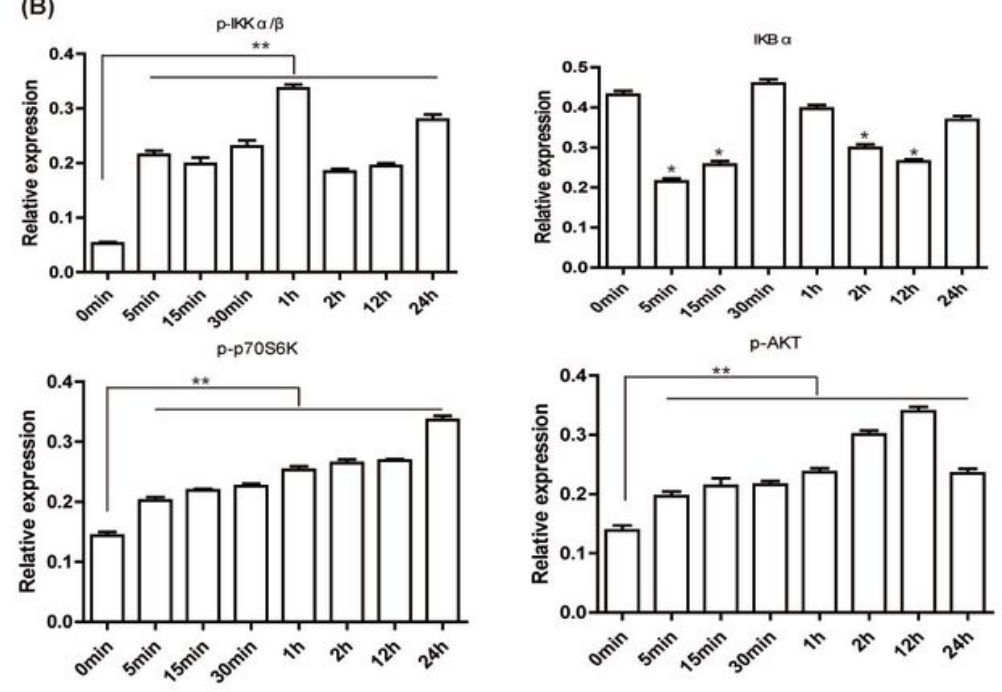

(D) TNF $\alpha \stackrel{\text { DMSO }}{-+} \frac{\text { RaPa }}{-+} \frac{\text { PP242 }}{-+}$

(E) DANCR
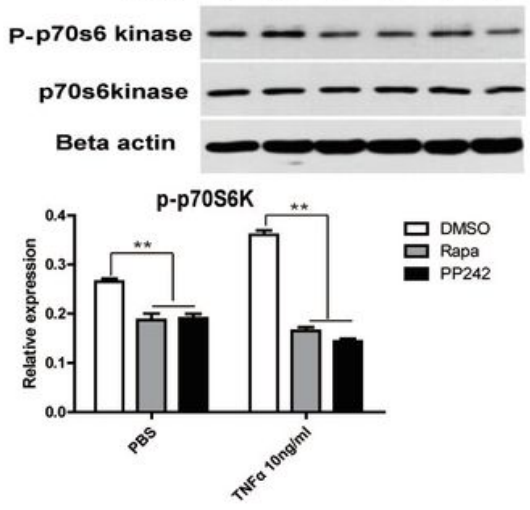

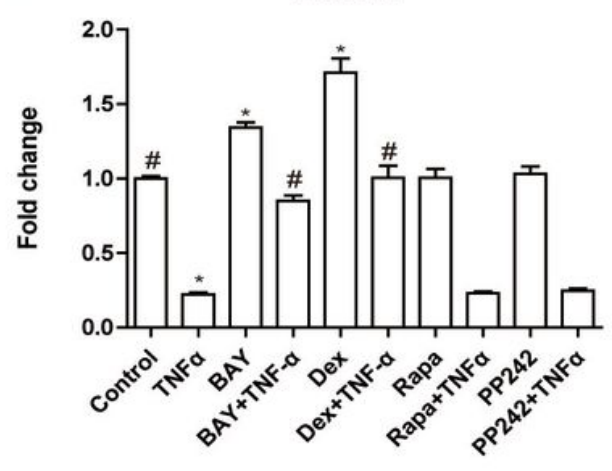

Figure 7

DANCR was involved in the NF-kB signaling pathway. (A) The effect of $10 \mathrm{ng} / \mathrm{ml}$ TNF-a on phosphorylated $1 \mathrm{IKKa} / \beta$, ІкBa, phosphorylated and pan p70s6 kinase, phosphorylated and pan AKT protein expression was assayed by WB analysis. B-actin was used as a loading control. (B) Densitometry assay with Image J. (C) The NF-KB inhibitors BAY and Dex inhibited TNF-a-induced activation of phospho- IKK a/ $\beta$. (D) The mTOR inhibitors Rapa and PP242 inhibited the TNF-a induced phosphorylation of p70s6 kinase. (E) DANCR expression was affected by NF-KB inhibitors BAY and Dex, while Rapa and PP242 had no effects. Results are presented as the mean $\pm S D\left(n=3,{ }^{*} p<0.05,{ }^{\star *} p<0.01\right.$, relative to control group; \#p<0.05, relative to TNF-a group). Abbreviations: BAY, BAY-117082; Dex, dexamethasone; Rapa, rapamycin. 